\title{
BWL für Juristen
}


Andreas Daum • Jürgen Petzold Matthias Pletke

BWL für Juristen

Eine praxisnahe Einführung in die betriebswirtschaftlichen Grundlagen

2. Auflage

Springer Gabler 
Prof. Dr. Andreas Daum

Fak. IV Wirtschaft

FH Hannover

Hannover, Deutschland

Dipl.Oec. Jürgen Petzold

Wilhelmshaven, Deutschland
Prof. Dr. Matthias Pletke

Fak. IV Wirtschaft

FH Hannover

Hannover, Deutschland

Die Deutsche Nationalbibliothek verzeichnet diese Publikation in der Deutschen Nationalbibliografie; detaillierte bibliografische Daten sind im Internet über http://dnb.d-nb.de abrufbar.

\section{Springer Gabler}

(C) Gabler Verlag | Springer Fachmedien Wiesbaden GmbH 2007, 2012

Das Werk einschließlich aller seiner Teile ist urheberrechtlich geschützt. Jede Verwertung, die nicht ausdrücklich vom Urheberrechtsgesetz zugelassen ist, bedarf der vorherigen Zustimmung des Verlags. Das gilt insbesondere für Vervielfältigungen, Bearbeitungen, Übersetzungen, Mikroverfilmungen und die Einspeicherung und Verarbeitung in elektronischen Systemen.

Die Wiedergabe von Gebrauchsnamen, Handelsnamen, Warenbezeichnungen usw. in diesem Werk berechtigt auch ohne besondere Kennzeichnung nicht zu der Annahme, dass solche Namen im Sinne der Warenzeichen- und Markenschutz-Gesetzgebung als frei zu betrachten wären und daher von jedermann benutzt werden dürften.

\section{Einbandentwurf: KünkelLopka GmbH, Heidelberg}

Gedruckt auf säurefreiem und chlorfrei gebleichtem Papier

Springer Gabler ist eine Marke von Springer DE.

Springer DE ist Teil der Fachverlagsgruppe Springer Science+Business Media

www.springer-gabler.de 


\section{Vorwort}

Interdisziplinarität gewinnt in einer globaler werdenden Wirtschaft zunehmend an Bedeutung. Dies gilt insbesondere für die Bereiche Betriebswirtschaft und Jura. Juristen und Betriebswirte müssen auf das sich ändernde Umfeld und die daraus abgeleiteten Chancen und Risiken vorbereitet sein, wollen sie im internationalen Wettbewerb erfolgreich sein. Immer häufiger wird der Jurist im Beruf mit der Beurteilung nationaler und internationaler betriebswirtschaftlicher Fallgestaltungen konfrontiert. Hierzu braucht er ein entsprechendes Grundlagenwissen.

Lehrbücher, die sich ausschließlich mit der Betriebswirtschaftslehre beschäftigen gibt es in großer Zahl. Leider gehen bisher nur sehr wenige Werke auf die speziellen Interessen der Juristin und des Juristen an der Schnittstelle zur Betriebswirtschaftslehre ein. An dieser Stelle ist eine Wissenslücke entstanden.

Die zweite Auflage von „BWL für Juristen“ soll diese Lücke schließen. Unser Ziel ist es, die notwendigen betriebswirtschaftlichen Grundlagen einfach und praxisnah zu vermitteln, so dass die Leserin und der Leser in die Lage versetzt werden, diese in ihrem Alltag gewinnbringend einzusetzen.

Wir wünschen allen Leserinnen und Lesern eine kurzweilige und gleichfalls gewinnbringende Lektüre. Über Rückmeldungen und Anmerkungen würden wir uns freuen.

\section{ANDREAS DAUM, JÜRGEN PETZOLD, MATTHIAS PLETKE}

andreas.daum@fh-hannover.de

juergen.petzold@hs-bremen.de

matthias.pletke@fh-hannover.de 


\section{Inhaltsverzeichnis}

Vorwort

1

Grundlagen der Betriebswirtschaftslehre 1

1.1

Betriebswirtschaftslehre aus der Sicht des Juristen..

Die Rechtspersönlichkeit

1.2.1 Der Betriebs- und Unternehmungsbegriff ......................................... 2

1.2.2 Die Unternehmung als Rechtssubjekt.............................................. 3

1.2.3 Die Einzelwirtschaft als Erfahrungsobjekt...................................... 6

1.2.4 Die Entscheidungen als Erkenntnisobjekt......................................... 9

1.3 Der Gegenstand der Betriebswirtschaftslehre.............................................. 11

1.3.1 Wechselwirkungen zu den Rechtswissenschaften und anderen Nachbardisziplinen

1.3.2 Unterscheidung zwischen Staat und Unternehmung Der Markt

1.3.3 Unterscheidung zwischen Güterproduktion und Dienstleistung 18

1.4 Erfolg durch Struktur - Wahl und Gestaltung der Unternehmungsform . 19

1.4.1 Formwechsel................................................................................... 20

1.4.2 Delegation ..................................................................................... 23

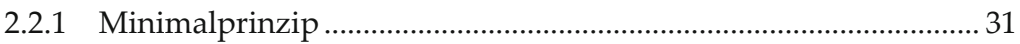

2.2.2 Maximalprinzip.................................................................... 31

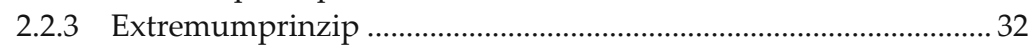

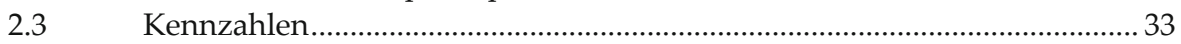

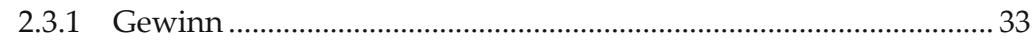

2.3.2 Wirtschaftlichkeit.......................................................................... 34



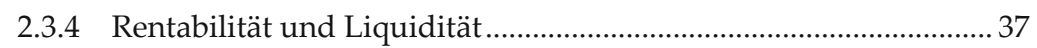

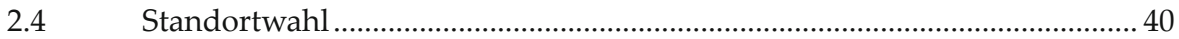

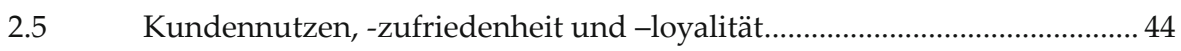

2.6 Qualität und Service als Erfolgspotentiale .................................................... 46

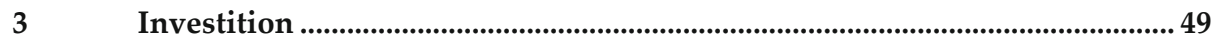

3.1 Begriff und Wesen von Investitionen ......................................................... 49

3.2 Verfahren der Investitions- und Wirtschaftlichkeitsrechnung ..................... 52 


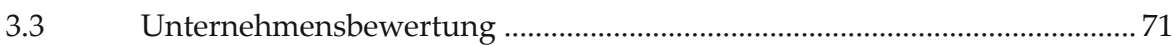

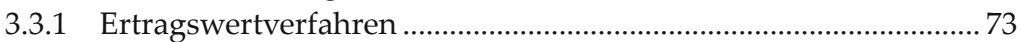

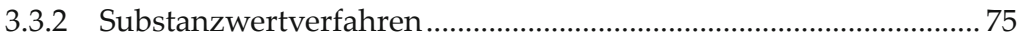

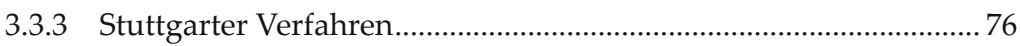

3.3.4 Discounted Cash Flow Verfahren..................................................... 77

3.4 Bewertungsbeispiel der BRAK ..................................................................... 79

4

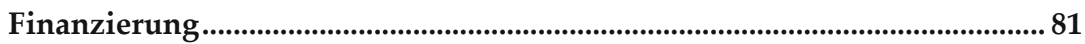

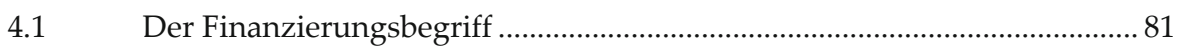

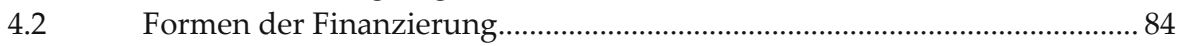

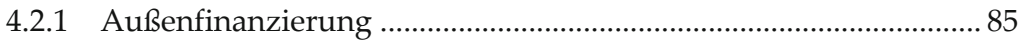

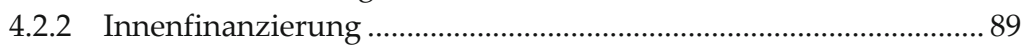

4.3 Steuerung der Liquidität mittels Finanzmittel (Finanzplanung) ................. 91

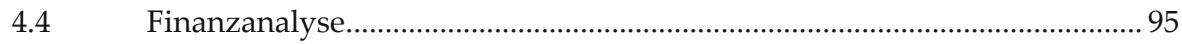

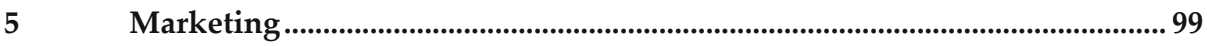

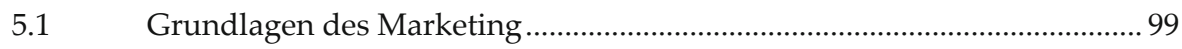

5.1.1 Begriff und Abgrenzung des Marktes ............................................ 99

5.1.2 Begriff und Merkmale des Marketing ........................................... 99

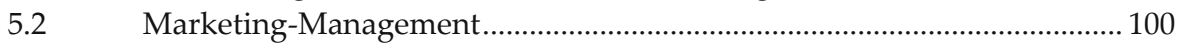

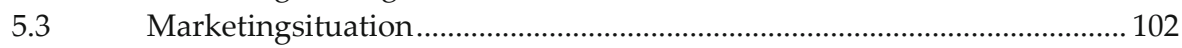

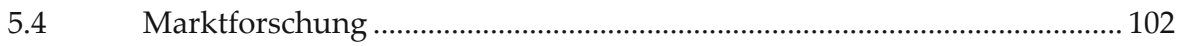

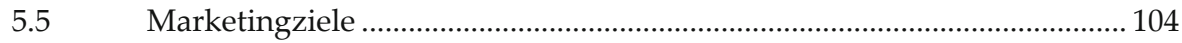

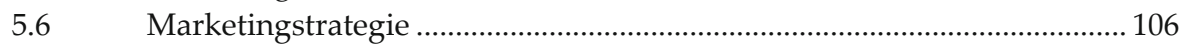

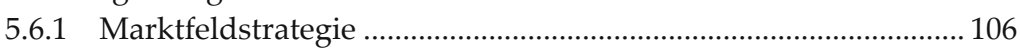

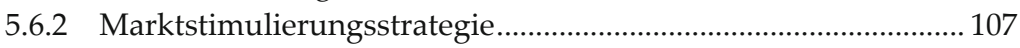

5.6.3 Marktparzellierungsstrategie..................................................... 107

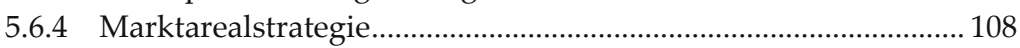

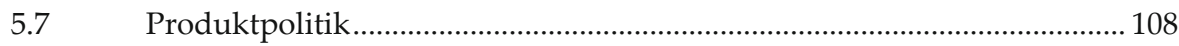

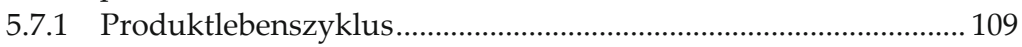

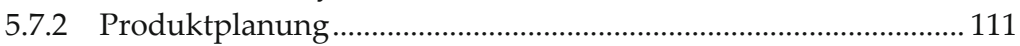

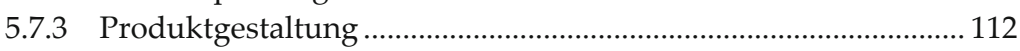

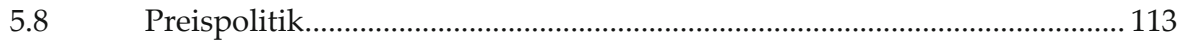

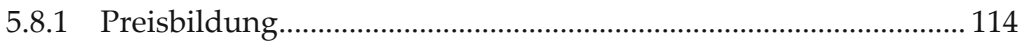

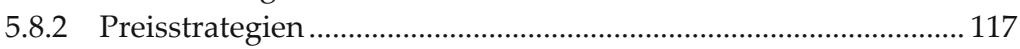

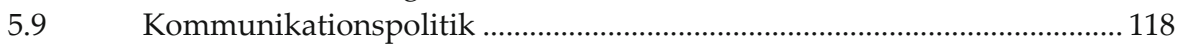

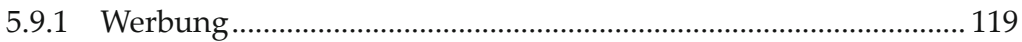



5.9.3 Öffentlichkeitsarbeit ........................................................................ 120

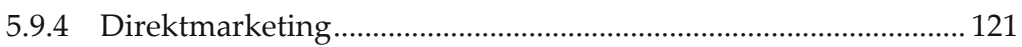

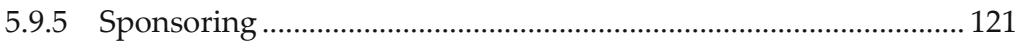

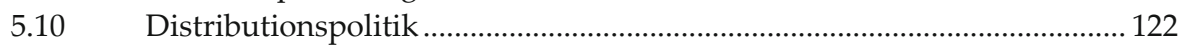

5.10.1 Akquisitorische Distribution........................................................ 122

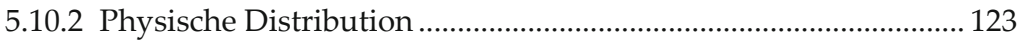




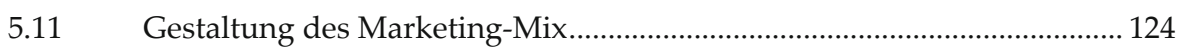

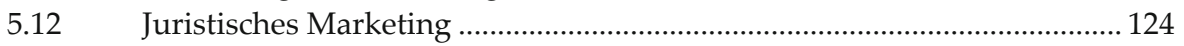

5.12.1 Anwaltliche Marketingkonzeption ….......................................... 125

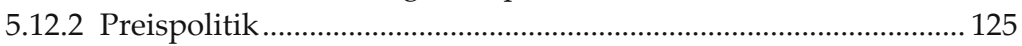

5.12.3 Produktpolitik ........................................................................... 125

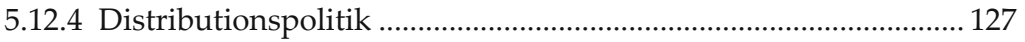

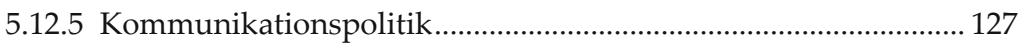

$6 \quad$ Teilgebiete des betrieblichen Rechungswesens ................................................ 131

6.1 Grundsätze ordnungsgemäßer Buchführung und Bilanzierung (GoB)... 131

6.2 Rechnungslegung (externes Rechnungswesen) ......................................... 138

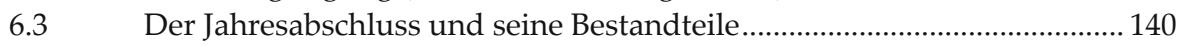

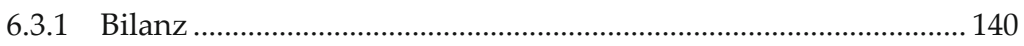

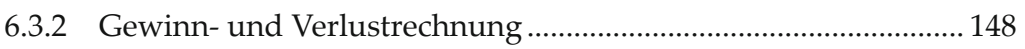

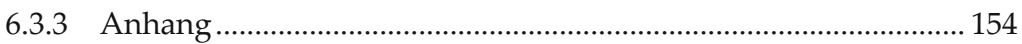

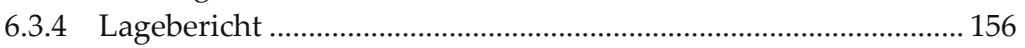

Internationale Konzernrechnungslegung …............................................... 157

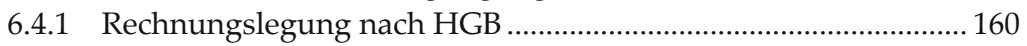

6.4.2 Rechnungslegung nach IAS ...................................................... 163

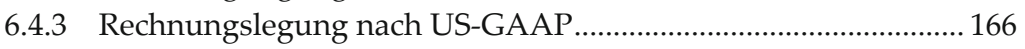

6.5 Bilanzpolitik und Probleme der Bilanzierungspraxis .................................. 168

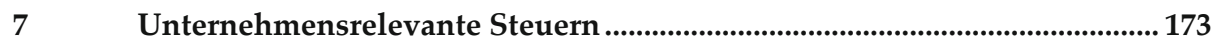

7.1 Grundlagen der steuerlichen Betrachtung …............................................... 173

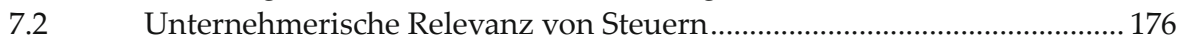

7.2.1 Steuereinfluss der Aufbauelemente eines Unternehmens ............ 177

7.2.2 Steuereinfluss der betriebswirtschaftlichen Funktionen ............. 178

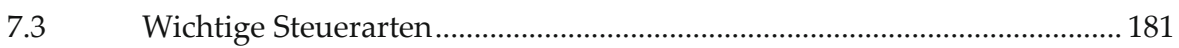

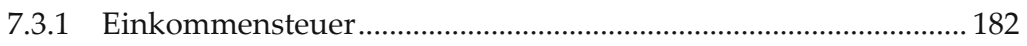

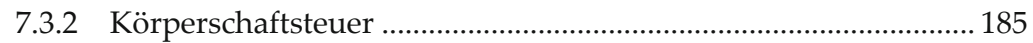

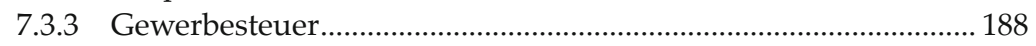

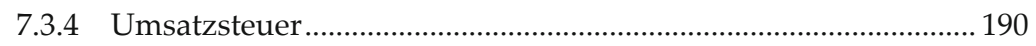

7.3.5 Ausgewählte Verbrauchs- und Verkehrssteuern .......................... 192

$8 \quad$ Kosten- und Leistungsrechnung............................................................................. 193

8.1 Kosten- und Leistungsrechnung - Zielsetzungen, Aufgaben, Definition 193

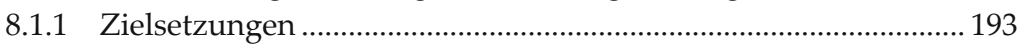

8.1.2 Aufgaben der Kosten- und Leistungsrechnung ............................ 194

8.1.3 Definition von Kosten und Leistungen .......................................... 196

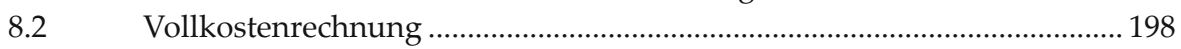

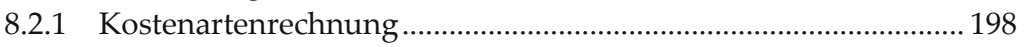

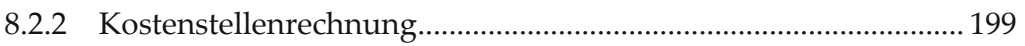

8.2.3 Kostenträgerrechnung …............................................................ 203 


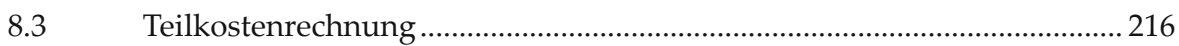

8.3.1 Einstufige Deckungsbeitragsrechnung...................................... 217

8.3.2 Mehrstufige Deckungsbeitragsrechnung ...................................... 218

8.3.3 Relative Deckungsbeitragsrechnung ........................................... 220

8.4 Moderne Kostenrechnungssysteme........................................................... 222

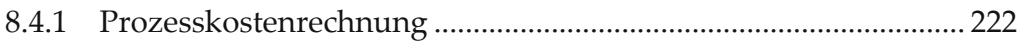

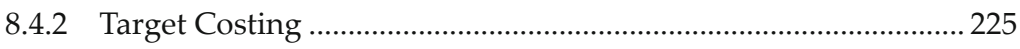

9

Controlling ..................................................................................................................... 227

9.1 Begriff, Einordnung und Bedeutung des Controllings für Juristen.......... 227

9.2 Strategisches versus operatives Controlling ................................................ 228



9.3.1 Controllingziele.......................................................................... 232

9.3.2 Controllingfunktion und -aufgaben .......................................... 233

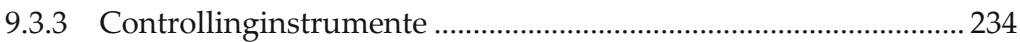

9.3.4 Controllingorganisation............................................................... 242

9.3.5 Aufgaben und Profil des Controllers.............................................. 242

9.4 Betriebswirtschaftliche Kennzahlen und Kennzahlensysteme................... 245

9.4.1 Kennzahlen und ihre Aussagen im Überblick............................... 245

9.4.2 Aufbau, Funktion und Aufgaben von Kennzahlensystemen...... 248

$10 \quad$ Unternehmensführung und Organisation............................................................. 251

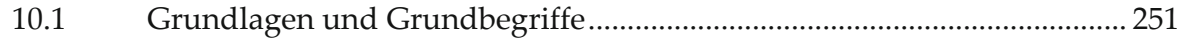

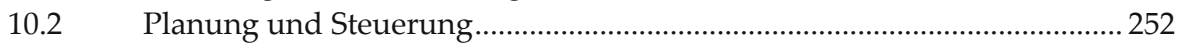

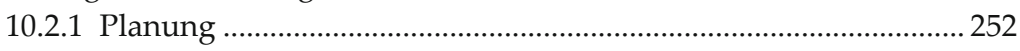



10.3 Strategisches Management..................................................................... 260

10.3.1 Unternehmensvision ..................................................................... 260

10.3.2 Unternehmensleitbild ................................................................ 262

10.3.3 Analyse der strategischen Ausgangsposition................................ 263

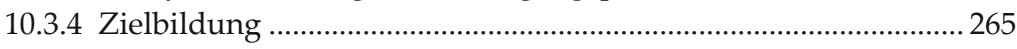

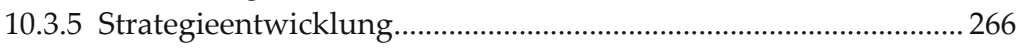

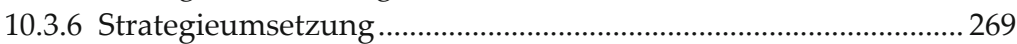

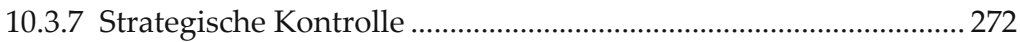

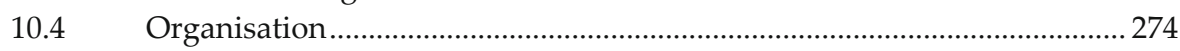

10.4.1 Aufbauorganisation..................................................................... 274

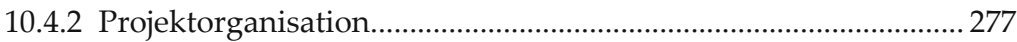

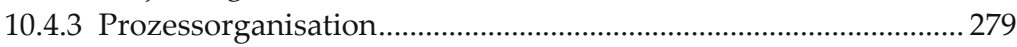

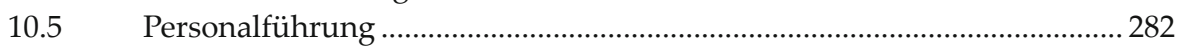

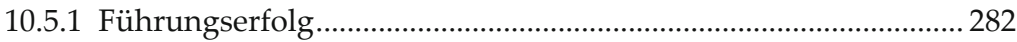

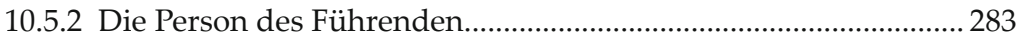

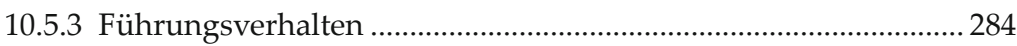

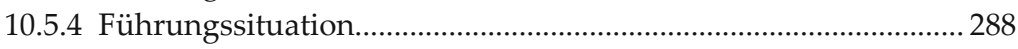

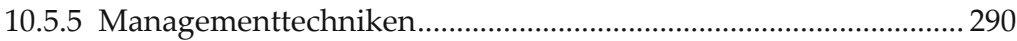




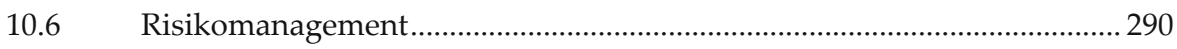

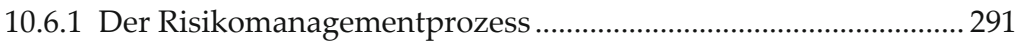

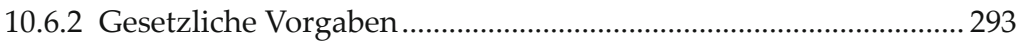

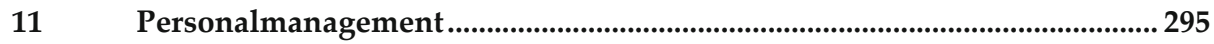

11.1 Ziele und Aufgaben des Personalmanagements........................................... 295

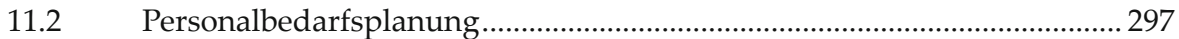

11.2.1 Quantitative Personalbedarfsermittlung....................................... 298

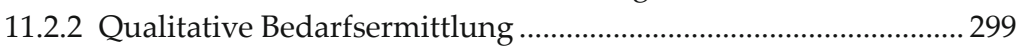



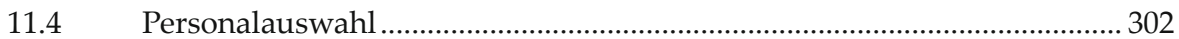

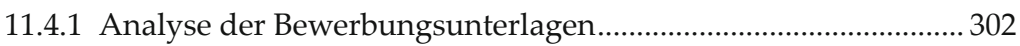

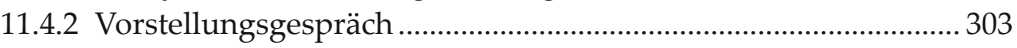

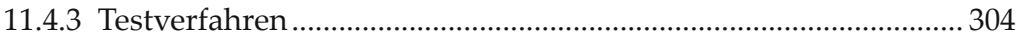

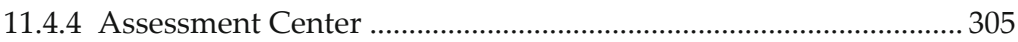

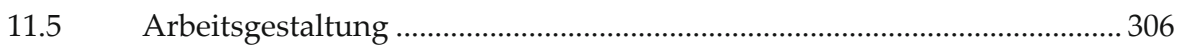

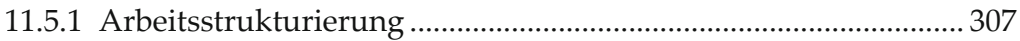

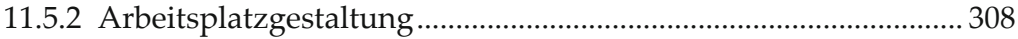

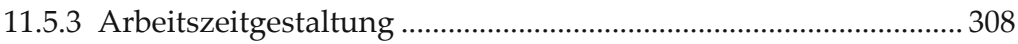

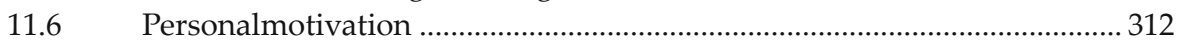

11.6.1 Beeinflussung des Leistungsverhaltens......................................... 312

11.6.2 Motivation und Anreize ................................................................... 314

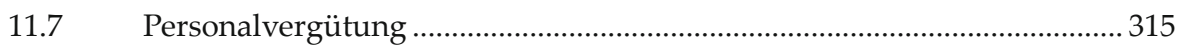

11.7.1 Potential-/Fähigkeitslohn ............................................................... 317

11.7.2 Arbeitswert-/Anforderungslohn ................................................. 317

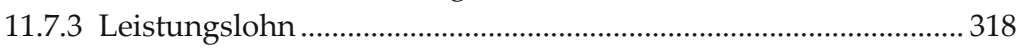

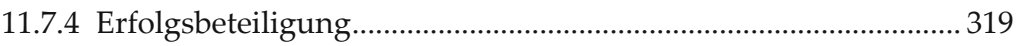

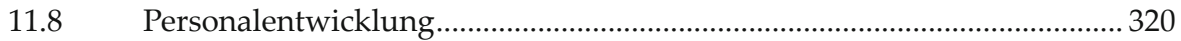

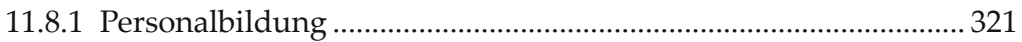

11.8.2 Prozess der systematischen Personalentwicklung........................ 323

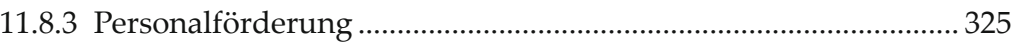

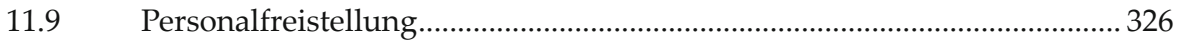

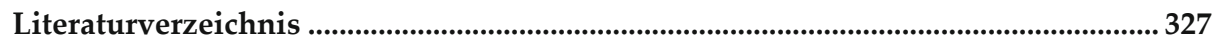

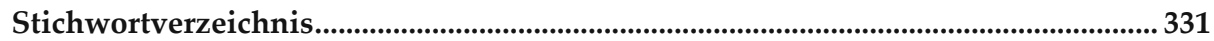

